\title{
Fuzzy e-paraopen Sets and Maps in Fuzzy Topological Spaces
}

\author{
M. Sankari and C. Murugesan
}

\begin{abstract}
This article is to study the concepts of fuzzy $e$-paraopen and fuzzy $e$-paraclosed sets in fuzzy topological spaces. Further, we extent to study few class of fuzzy maps namely fuzzy $e$-paracontinuous, $*$-fuzzy $e$-paracontinuous, fuzzy $e$-parairresolute, fuzzy minimal $e$-paracontinuous, fuzzy maximal $e$-paracontinuous mappings and study their properties.
\end{abstract}

\section{Index Terms}

Fuzzy $e$-paraopen, fuzzy $e$-paracontinuous, fuzzy minimal $e$-paracontinuous, fuzzy maximal $e$-paracontinuous.

\section{INTRODUCTION}

Zadeh [10] established fuzzy sets and since fuzzy topology developed by Chang [2]. The notions of fuzzy minimal(maximal) open and paraopen sets respectively explored by Ittanagi and Wali in [3] and [4]. Subsequently Mukherjee and Bagchi in [1] introduced and showed the notion of mean open set. In section II of current article we introduce the perception of fuzzy $e$-paraopen set and investigate some comparative results. In section III, we introduce fuzzy $e$-paracontinuous, $*$-fuzzy $e$-paracontinuous, fuzzy $e$-parairresolute, fuzzy minimal $e$-paracontinuous, fuzzy maximal $e$-paracontinuous maps and from which we investigate some results with appropriate examples. Throughout this paper following terminologies "fuzzy e-open, fuzzy e-paraopen, fuzzy e-paraclosed, fuzzy minimal e-open, fuzzy minimal e-closed, fuzzy maximal e-open, fuzzy maximal eclosed are respectively abbreviated as Fe-O,Fe-PO,Fe-PC,FMIe-O,FMIe-C,FMAe-O,FMAe-C respectively. Throught this paper $F$ and $Y$ stands for fuzzy topological spaces."

The following terminologies "fuzzy e-continuous, fuzzy e-paracontinuous, fuzzy minimal e-continuous,fuzzy maximal econtinuous, fuzzy minimal e-paracontinuous, fuzzy maximal e-paracontinuous, fuzzy maximal e-parairresolute are respectively abbreviated as f.e-c,f.e-pc,f.mi.e-c,f.ma.e-c,f.mi.e-pc,f.ma.e-pc,f.mi.e-p.i,f.ma.e-p.i respectively"

Definition 1.1 A fuzzy subset $\xi$ of a space $F$ is called fuzzy regular open [3] (resp. fuzzy $\operatorname{regular} \operatorname{closed})$ if $\xi=\operatorname{Int}(\mathrm{Cl}(\xi)$ ) $(\operatorname{resp} . \xi=\mathrm{Cl}(\operatorname{Int}(\xi)))$.

The fuzzy $\delta$-interior of a fuzzy subset $\xi$ of $F$ is the union of all fuzzy regular open sets contained in $\xi$. A fuzzy subset $\xi$ is called fuzzy $\delta$-open [9] if $\xi=\operatorname{Int}_{\delta}(\xi)$. The complement of fuzzy $\delta$-open set is called fuzzy $\delta$-closed (i.e., $\xi=\mathrm{Cl}_{\delta}(\xi)$ ).

Definition 1.2 A fuzzy subset $\xi$ of a fts $F$ is called fuzzy $e$-open [8] if $\left.\xi \leq \operatorname{cl}_{\left(i^{\prime}\right.} \xi\right) \cup$ int $\left(\mathrm{cl}_{\delta} \xi\right)$ and fuzzy $e$-closed set if $\xi \geq \operatorname{cl}\left(\operatorname{int}_{\delta} \xi\right) \cap \operatorname{int}\left(\mathrm{cl}_{\delta} \xi\right)$.

Definition 1.3 [7]A proper nonzero fuzzy $e$-open set $\alpha$ of $F$ is said to be a

(i)fuzzy minimal $e$-open if $1_{F}$ and $\alpha$ are only fuzzy $e$-open sets contained in $\alpha$.

(ii)fuzzy maximal $e$-open $1_{\digamma}$ and $\alpha$ are only fuzzy $e$-open sets containing $\alpha$.

Definition 1.4 A map from fts $F$ to another fts $Y$ is called,

(i) fuzzy minimal $e$-continuous[7] if $f^{-1}(\lambda)$ is a fuzzy $e$-open set on $F$ for any fuzzy minimal $e$-open set $\lambda$ on $Y$.

(ii)fuzzy maximal $e$-continuous[7] if $f^{-1}(\lambda)$ is a fuzzy $e$-open set on $F$ for any fuzzy maximal $e$-open set $\lambda$ on $Y$.

\section{Fuzzy $e$-Paraopen ANd Some of their Properties}

Definition 2.1 A Fe-O set $\beta$ of a fts $F$ is said to be a Fe-PO set if is neither FMIe-O nor FMAe-O set.

The complement of Fe-PO set is Fe-PC set.

Remark 2.2 It could be clear from definitions that every Fe-PO set is a Fe-O set and every Fe-PC set is a Fe-C set converse is not true as shown in the succeeding example.

Example 2.3 Let $\beta_{1}, \beta_{2}, \beta_{3}$ and $\beta_{4}$ be fuzzy sets on $F=\{a, b, c\}$. Then $\beta_{1}=\frac{0.5}{a}+\frac{0.8}{b}+\frac{0.8}{c}, \beta_{2}=\frac{0.5}{a}+\frac{0.8}{b}+\frac{0.9}{c} \beta_{3}=\frac{1.0}{a}+\frac{0.9}{b}+\frac{0.8}{c}$ and $\beta_{4}=\frac{1.0}{a}+\frac{0.9}{b}+\frac{0.9}{c}$ be fuzzy sets with $\mathfrak{\mho}_{1}=\left\{0_{F}, \beta_{1}, \beta_{2}, \beta_{3}, \beta_{4}, 1_{F}\right\}$, Then $F M_{i} O(F)=\left\{\beta_{1}\right\}, F M_{a} O(F)=\left\{\beta_{4}\right\}, F M_{i} C(F)=\left\{\beta_{4}^{c}\right\}$, $F M_{a} C(F)=\left\{\beta_{1}^{c}\right\}, F P_{a} O(F)=\left\{\beta_{2}, \beta_{3}\right\}, F P_{a} C(F)=\left\{\beta_{2}^{c}, \beta_{3}^{c}\right\}$. Here $\beta_{1}$ is a Fe-O set but not a Fe-PO set and $\beta_{4}^{c}$ is a fuzzy $e$-closed set but not a Fe-PC set.

ISSN: 2736-5484

DOI: http://dx.doi.org/10.24018/ejmath.2022.3.1.83

Published on February 15, 2022.

M. Sankari is with Department of Mathematics, Lekshmipuram College of Arts and Science,Neyyoor, Kanyakumari, Tamil Nadu-629 802, India. (e-mail: sankarisaravanan1968@gmail.com)

C. Murugesan is Research Scholar, Pionneer Kumaraswami College of Arts and Science,Nagercoil, Tamil Nadu-629 003, India.(Affiliated to Manonmaniam Sundaranar University, Tirunelvelli. (e-mail: kumarithozhanmurugesan@gmail.com). 
Remark 2.4 The succeeding example revealed that union and intersection of Fe-PO (resp. Fe-PC) sets need not be a Fe-PO (resp. Fe-PC).

Example 2.5 In example 2.3, fuzzy sets $\beta_{2}, \beta_{3}$ are Fe-PO sets but $\beta_{2} \vee \beta_{3}=\beta_{4}$ and $\beta_{2} \wedge \beta_{3}=\beta_{1}$ which are not Fe-PO sets. Similarly for the Fe-PC sets $\beta_{2}^{c}, \beta_{3}^{c}$ but $\beta_{2}^{c} \vee \beta_{3}^{c}=\beta_{1}^{c}$ and $\beta_{2}^{c} \wedge \beta_{3}^{c}=\beta_{4}^{c}$ which are not Fe-PC sets.

Theorem 2.6 Let $\alpha$ be a nonzero proper Fe-PO subset of $F$. Then there exists a FMIe-O set $\beta$ such that $\beta<\alpha$.

Proof. Since the definition of FMIe-O set, we can conclude that $\beta<\alpha$.

Theorem 2.7 Let $\alpha$ be a nonzero proper Fe-PO subset of $F$. Then there exists a FMAe-O set $\mathcal{P}$ such that $\alpha<\mathcal{P}$.

Proof. Since the definition of FMAe-O set, we can conclude that $\alpha<\mathcal{P}$.

Theorem 2.8 (i)Let $\alpha$ be a Fe-PO and $\beta$ be a FMIe-O set in $F$. Then $\alpha \wedge \beta=0_{F}$ or $\beta<\alpha$. (ii)i)Let $\alpha$ be a Fe-PO and $\tau_{1}$ be a FMAe-O set in $F$. then $\alpha \vee \tau_{1}=1_{F}$ or $\alpha<\tau_{1}$.

(iii)Intersection of Fe-PO sets is either Fe-PO or FMIe-O set.

Proof. (i) Let $\alpha$ be a Fe-PO and $\beta$ be a FMIe-O set in $F$. Then $\alpha \wedge \beta=0_{F}$ or $\alpha \wedge \beta \neq 0_{F}$. Suppose $\alpha \wedge \beta=0_{F}$, then we need not prove anything. Assume $\alpha \wedge \beta \neq 0_{F}$. Then we get $\alpha \wedge \beta$ is a Fe-O set and $\alpha \wedge \beta<\beta$. Hence $\beta<\alpha$.

(ii) Let $\alpha$ be a Fe-PO and $\gamma$ be a FMAe-O set in $F$. Then $\alpha \vee \gamma=1_{F}$ or $\alpha \vee \beta \neq 1_{F}$. Assume $\alpha \vee \gamma=1_{F}$, then we need not prove anything. Suppose $\alpha \vee \gamma \neq 1_{F}$. Then we get $\alpha \vee \gamma$ is a Fe-O set and $\gamma<\alpha \vee \gamma$. Since $\gamma$ is a FMAe-O set, $\alpha \vee \gamma=\gamma$ which implies $\alpha<\gamma$.

(iii)Let $\alpha$ and $\eta$ be a Fe-PO sets in $F$. As $\alpha \wedge \eta$ is a Fe-PO set then we need not prove anything. Assume $\alpha \wedge \eta$ is not a Fe-PO set. Since definition, $\alpha \wedge \eta$ is a FMIe-O or FMAe-O set. If $\alpha \wedge \eta$ is a f.mi. $e$-open set then we need not prove anything. Suppose $\alpha \wedge \eta$ is a FMAe-O set. Now $\alpha \wedge \eta<\alpha$ and $\alpha \wedge \eta<\eta$ which contradicts the fact that $\alpha$ and $\eta$ are Fe-PO sets. Therefore $\alpha \wedge \eta$ is not a FMAe-O set. That is $\alpha \wedge \eta$ must be a FMIe-O set.

Theorem 2.9 A subset $\tau_{1}$ of $F$ is Fe-PC iff it is neither FMAe-C nor FMIe-C set.

Proof. Since the definition of FMAe-C set and the fact that the complement of FMIe-O set is FMAe-C set and the complement of FMAe-O set is FMIe-C set.

Theorem 2.10 Let $F$ be a fts and $\tau_{1}$ be a nonzero Fe-PC subset of $F$. Then there exists a f.mi.e-c set $\mathcal{P}$ such that $\mathcal{P}<\tau_{1}$.

Proof. Since the definition of FMIe-C set we can conclude that $\mathcal{P}<\tau_{1}$.

Theorem 2.11 Let $F$ be a fts and $\tau_{1}$ be a nonzero Fe-PC subset of $F$. Then there exists a f.ma. closet set $Q$ such that $\tau_{1}<Q$.

Proof. Since the definition of FMAe-C set we can conclude that $\tau_{1}<Q$.

Theorem 2.12 Let $F$ be a fts. (i)Let $\delta$ be a Fe-PC and $\tau$ be a FMIe-C set. Then $\delta \wedge \tau=0_{F}$ or $\tau<\delta$.

(ii)Let $\delta$ be a Fe-PC and $\gamma$ be a FMAe-C set. Then $\delta \vee \gamma=1_{F}$ or $\delta<\gamma$.

(iii)Intersection of Fe-PC sets is either Fe-PC or FMIe-C set.

Proof. (i) Let $\delta$ be a Fe-PC and $\tau$ be a FMIe-C set in $F$. Then $\left(1_{F}-\delta\right)$ is Fe-PO and $\left(1_{F}-\tau\right)$ is FMAe-O set in $F$. By Theorem 2.8(ii) we have $\left(1_{F}-\delta\right) \vee\left(1_{F}-\tau\right)=F$ or $\left(1_{F}-\delta\right)<\left(1_{\digamma}-\tau\right)$ which implies $1_{F}-(\delta \wedge \tau)=1_{\digamma}$ or $\tau<\delta$. Therefore $\delta \wedge \tau=0_{F}$ or $\tau<\delta$.

(ii) Let $\delta$ be a Fe-PC and $\gamma$ be a FMAe-C set in $F$. Then $\left(1_{F}-\delta\right)$ is Fe-PO and $\left(1_{F}-\gamma\right)$ is FMIe-O sets in $F$. By Theorem 2.8(i) we have $\left(1_{F}-\delta\right) \wedge\left(1_{F}-\gamma\right)=0_{F}$ or $1_{F}-\gamma<1_{F}-\delta$ which implies $1_{F}-(\delta \vee \gamma)=0_{F}$ or $\delta<\gamma$. Therefore $\delta \vee \gamma=1_{F}$ or $\delta<\gamma$.

(iii)Let $\delta$ and $\eta$ be a Fe-PC sets in $F$. As $\delta \wedge \eta$ is a Fe-PC set then nothing to prove. Assume $\delta \wedge \eta$ is not a Fe-PC set. By definition, $\delta \wedge \eta$ is a FMIe-C or FMAe-C set. If $\delta \wedge \eta$ is a f.mi. $e$-closed set, then nothing to prove. Suppose $\delta \wedge \eta$ is a FMAe-C set. Now $\delta<\delta \wedge \eta$ and $\eta<\delta \wedge \eta$ which contradicts the fact that $\delta$ and $\eta$ are Fe-PC sets. Therefore $\delta \wedge \eta$ is not a FMAe-C set. That is $\delta \wedge \eta$ must be a FMIe-C set. 


\section{Fuzzy e-paracontinuous Maps and Some of Their Properties}

Definition 3.1 A map $\psi$ from fts $F$ to another fts $\Delta$ is called

(i) f.e-pc if $\psi^{-1}(\alpha)$ is a Fe-O set on $F$ for every Fe-PO set $\alpha$ on $\Delta$.

(ii) $*$-f.e-pc if $\psi^{-1}(\alpha)$ is a Fe-PO set on $F$ for every Fe-O set $\alpha$ on $\Delta$.

(iii) f.e-p.i if $\psi^{-1}(\alpha)$ is a Fe-PO set on $F$ for every Fe-PO set $\alpha$ on $\Delta$.

(iv) f.mi.e-pc if $\psi^{-1}(\alpha)$ is a Fe-PO set on $F$ for every FMIe-O set $\alpha$ on $\Delta$.

(v) f.ma.e-pc if $\psi^{-1}(\alpha)$ is a Fe-PO set on $F$ for every FMAe-O set $\alpha$ on $\Delta$.

Theorem 3.2 Every f.e-c map is f.e-pc but not conversely.

Proof. Let $\psi: F \rightarrow \Delta$ be a f.e-c map. We have to prove $\psi$ is f.e-pc. Let $\alpha$ be any Fe-PO set in $\Delta$. Since every Fe-PO set is a Fe-O set, $\alpha$ is Fe-O set in $\Delta$. Since $\psi$ is a f.e-c, $\psi^{-1}(\alpha)$ is Fe-O set in F. Hence $\psi$ is a f.e-pc.

Example 3.3 Let $\alpha_{1}, \alpha_{1}{ }^{c}, \alpha_{2}, \alpha_{3}, \alpha_{4}$ and $\alpha_{5}$ be fuzzy sets on $F=\{a, b, c\}$ with

$\alpha_{1}=\frac{0.3}{a}+\frac{0.4}{b}+\frac{0.4}{c}, \alpha_{2}=\frac{0.3}{a}+\frac{0.4}{b}+\frac{0.5}{c}, \alpha_{3}=\frac{0.6}{a}+\frac{0.5}{b}+\frac{0.4}{c}, \alpha_{4}=\frac{0.6}{a}+\frac{0.5}{b}+\frac{0.5}{c}, \alpha_{5}=\frac{0.7}{a}+\frac{0.6}{b}+\frac{0.4}{c}$ and $\alpha_{1}{ }^{c}=\frac{0.7}{a}+\frac{0.6}{b}+\frac{0.5}{c}$.

Let $\tau_{1}=\left\{0_{F}, \alpha_{1}, \alpha_{2}, \alpha_{3}, \alpha_{4}, 1_{F}\right\}$ and $\tau_{2}=\left\{0_{F}, \alpha_{1}, \alpha_{1}{ }^{c}, \alpha_{2}, \alpha_{3}, \alpha_{4}, \alpha_{5}, 1_{F}\right\}$ be fuzzy topologies on $F$. Consider the fuzzy identity mapping $\psi:\left(F, \tau_{1}\right) \rightarrow\left(F, \tau_{2}\right)$. Then $\psi$ is f.e-pc but not f.e-c mapping because for a Fe-O set $\alpha_{5}$ on $\left(F, \tau_{2}\right), \psi^{-1}\left(\alpha_{5}\right)=\alpha_{5}$ which is not a Fe-O set on $\left(F, \tau_{1}\right)$.

Theorem 3.4 Every $*$-f.e-pc is f.e-c but not conversely.

Proof. Let $\psi: F \rightarrow \Delta$ be a $*$-f.e-pc map. We have to prove $\psi$ is f.e-c. Let $\alpha$ be a Fe-O set in $\Delta$. Since $\psi$ is $*$-f.e-pc, $\psi^{-1}(\alpha)$ is $\mathrm{Fe}-\mathrm{PO}$ set in $F$. Since every Fe-PO set is a Fe-O set, $\psi^{-1}(\alpha)$ is Fe-O set in $F$. Hence $\psi$ is a f.e-c.

Example 3.5 Let $\beta_{1}, \beta_{2}$ and $\beta_{3}$ be fuzzy sets on $F=\Delta=\{a, b, c\}$. Then $\beta_{1}=\frac{1.0}{a}+\frac{0.0}{b}+\frac{0.0}{c}, \beta_{2}=\frac{1.0}{a}+\frac{0.6}{b}+\frac{0.0}{c}$ and $\beta_{3}=\frac{1.0}{a}+\frac{0.6}{b}+\frac{0.5}{c}$ are defined as follows: Consider $\mathfrak{F}_{1}=\left\{0_{F}, \beta_{1}, \beta_{2}, \beta_{3}, 1_{F}\right\}$, Let $\psi: F \rightarrow \Delta$ be an identity mapping. Then $\psi$ is f.e-c but not $*$-f.e-pc mapping since for the Fe-O set $\beta_{3}$ on $\Delta, \psi^{-1}\left(\beta_{3}\right)=\beta_{3}$ which is not a Fe-PO set on $F$.

Theorem 3.6 Every $*$-f.e-pc is f.e-pc but not conversely.

Proof. The proof follows from Theorems 3.2 and 3.4.

Example 3.7 In Example 3.5, " $\psi$ is f.e-pc map but it is not $*$-f.e-pc map."

Theorem 3.8 Every f.e-p.i map is f.e-pc but not conversely.

Proof. Let $\psi: F \rightarrow \Delta$ be a f.e-p.i map. We have to prove that $\psi$ is f.e-pc. Let $\alpha$ be any Fe-PO set in $\Delta$. Since $\psi$ is f.e-p.i, $\psi^{-1}(\alpha)$ is Fe-PO set in F. Since every Fe-PO set is a Fe-O set, $\psi^{-1}(\alpha)$ is Fe-O set in $F$. Hence $\psi$ is a f.e-pc map.

Example 3.9 As described in Example 3.5, consider $\mathfrak{F}_{3}=\left\{0_{F}, \beta_{2}, \beta_{3}, 1_{F}\right\}$ and $\mathfrak{F}_{1}=\left\{0_{\Delta}, \beta_{1}, \beta_{2}, \beta_{3}, 1_{\Delta}\right\}$. Let $\psi: F \rightarrow \Delta$ be an identity mapping. Then $\psi$ is f.e-pc but not f.e-p.i mapping since for the Fe-PO set $\beta_{2}$ on $\Delta, \psi^{-1}\left(\beta_{2}\right)=\beta_{2}$ which is not a Fe-PO set on $F$.

Theorem 3.10 Every $*$-f.e-pc is f.e-p.i but not conversely.

Proof. Let $\psi: F \rightarrow \Delta$ be a f.e-pc map. We have to prove that $\psi$ is f.e-p.i. Let $\alpha$ be a Fe-PO set in $\Delta$. Since every Fe-PO set is a Fe-O set, $\alpha$ is a Fe-O set. Since $\psi$ is $*$-f.e-pc, $\psi^{-1}(\alpha)$ is Fe-PO set in $F$. Hence $\psi$ is a f.e-p.i map.

Example 3.11 In Example 3.5," $\psi$ is f.e-p.i map but it is not $*$-f.e-pc map."

Remark 3.12 Fuzzy e-p.irresolute and f.e-c maps are independent of each other.

Example 3.13In Example 3.3, $\psi$ is f.e-p.i map but it is not f.e-c map because for the Fe-O set $\beta_{5}$ on $\Delta, \psi^{-1}\left(\beta_{5}\right)=\beta_{5}$ which is not a $\mathrm{Fe}-\mathrm{O}$ set on $F$.

Let $\beta_{1}, \beta_{2}, \beta_{3}$ be fuzzy sets on $F=\{a, b, c\}$ and let $\alpha_{1}, \alpha_{2}, \alpha_{3}$ be fuzzy sets on $\Delta=\{x, y, z\}$. Then $\beta_{1}=\frac{0.2}{a}+\frac{0.2}{b}+\frac{0.2}{c}$, $\beta_{2}=\frac{0.3}{a}+\frac{0.3}{b}+\frac{0.3}{c}, \beta_{3}=\frac{0.7}{a}+\frac{0.7}{b}+\frac{0.7}{c}, \alpha_{1}=\frac{0.2}{x}+\frac{0.0}{y}+\frac{0.2}{z}, \alpha_{2}=\frac{0.7}{x}+\frac{0.0}{y}+\frac{0.7}{z}, \alpha_{3}=\frac{0.7}{x}+\frac{0.7}{y}+\frac{0.7}{z}$ are defined as follows: Consider $\mathfrak{F}_{1}=\left\{0_{F}, \beta_{1}, \beta_{2}, \beta_{3}, 1_{F}\right\}, \mathfrak{F}_{2}=\left\{0_{\Delta}, \alpha_{1}, \alpha_{1}, \alpha_{3}, 1_{\Delta}\right\}$. Let $\psi: F \rightarrow \Delta$ be a fuzzy mapping defined as $f(a)=f(b)=f(c)=y$. Then $\psi$ is f.e-c but not fuzzy $e$-parairreolute because for the Fe-PO set $\alpha_{2}$ on $\Delta, \psi^{-1}\left(\alpha_{3}\right)=0_{F}$ which is not a Fe-PO set on $F$.

Theorem 3.14 Every f.mi.e-pc map is f.mi. $e$-continuous but not conversely.

Proof. Let $\psi: F \rightarrow \Delta$ be a f.mi.e-pc map. We have to prove that $\psi$ is f.mi. $e$-continuous. Let $\tau_{1}$ be any FMIe-O set in $\Delta$. Since $\psi$ is f.mi.e-pc, $\psi^{-1}\left(\tau_{1}\right)$ is Fe-PO set in $F$. Since every Fe-PO set is a Fe-O set, $\psi^{-1}\left(\tau_{1}\right)$ is a Fe-O set in $F$. Hence $\psi$ is a fuzzy minimal $e$-continuous.

Example 3.15From Example 3.2, $\psi$ is f.mi. $e$-continous but it is not a f.mi. $e$-p.continuous, since for the FMIe-O $\beta_{1}$ on $\Delta$, $\psi^{-1}\left(\beta_{1}\right)=\beta_{1}$ which is not a Fe-PO set on $F$.

Remark 3.16Fuzzy minimal $e$-p.continuous and f.e-pc(resp. f.e-c) are independent of each other.

Example 3.17 Let $\beta_{1}, \beta_{2}$ be fuzzy sets on $F=\{a, b, c\}$ and let $\alpha_{1}, \alpha_{2}, \alpha_{3}$ be fuzzy sets on $\Delta=\{x, y, z\}$. Then $\beta_{1}=\frac{0.5}{a}+\frac{0.0}{b}+\frac{0.0}{c}$, $\beta_{2}=\frac{0.5}{a}+\frac{0.7}{b}+\frac{0.0}{c}, \beta_{3}=\frac{0.5}{a}+\frac{0.7}{b}+\frac{0.1}{c}, \alpha_{1}=\frac{0.5}{x}+\frac{0.7}{y}+\frac{0.0}{z}, \alpha_{2}=\frac{0.5}{x}+\frac{0.7}{y}+\frac{0.9}{z}, \alpha_{3}=\frac{0.5}{x}+\frac{0.8}{y}+\frac{0.0}{z}$ and $\alpha_{4}=\frac{0.5}{x}+\frac{0.8}{y}+\frac{0.9}{z}$ are 
defined as follows: Consider $\mathfrak{F}_{1}=\left\{0_{F}, \beta_{1}, \beta_{2}, \beta_{3}, 1_{F}\right\}, \widetilde{F}_{2}=\left\{0_{\Delta}, \alpha_{1}, \alpha_{1}, \alpha_{3}, \alpha_{4}, 1_{\Delta}\right\}$. Let $\psi: F \rightarrow \Delta$ be an identity maping. Then $\psi$ is f.mi.e-pc but not f.e-pc(resp. f.e-c) map because for the Fe-PO set $\alpha_{3}$ on $\Delta, \psi^{-1}\left(\alpha_{3}\right)=\alpha_{3}$ which is not a Fe-O set on $F$. In Example 3.2, $\psi$ is f.e-pc but not f.mi.e-pc.

Theorem 3.18 Every f.ma.e-pc is f.ma.e-c but not conversely.

Proof. Let $\psi: F \rightarrow \Delta$ be a f.ma.e-pc map. To prove $\psi$ is f.mi. $e$-continuous. Let $\delta$ be any FMAe-O set in $\Delta$. Since $\psi$ is f.ma.e-pc, $\psi^{-1}(\delta)$ is Fe-PO set in $F$. Since every Fe-PO set is a Fe-O set, $\psi^{-1}(\delta)$ is a Fe-O set in $F$. Hence $\psi$ is a f.ma.e-c.

Example 3.19 In Example 3.2, " $\psi$ is f.ma.e-c but it is not f.ma.e-pc map."

Remark 3.20 Fuzzy maximal $e$-p.continuous and f.e-pc(resp. f.e-c) are independent of each other.

Example 3.21 Let $\beta_{1}, \beta_{2}$ be fuzzy sets on $F=\{a, b, c, d\}$ and let $\alpha_{1}, \alpha_{2}, \alpha_{3}$ be fuzzy sets on $\Delta=\{x, y, z, w\}$. Then $\beta_{1}=$ $\frac{0.0}{a}+\frac{0.0}{b}+\frac{0.0}{c}+\frac{0.9}{d}, \beta_{2}=\frac{0.0}{a}+\frac{0.0}{b}+\frac{0.7}{c}+\frac{0.9}{d}, \beta_{3}=\frac{0.0}{a}+\frac{0.5}{b}+\frac{0.7}{c}+\frac{0.9}{d}, \beta_{4}=\frac{0.2}{a}+\frac{0.5}{b}+\frac{0.7}{c}+\frac{0.9}{d}, \alpha_{1}=\frac{0.0}{x}+\frac{0.0}{y}+\frac{0.3}{z}+\frac{0.0}{w}$, $\alpha_{2}=\frac{0.0}{x}+\frac{0.0}{y}+\frac{0.3}{z}+\frac{0.9}{w}, \alpha_{3}=\frac{0.0}{x}+\frac{0.5}{y}+\frac{0.7}{z}+\frac{0.9}{w}$, are defined as follows: Consider $\mathfrak{F}_{1}=\left\{0_{F}, \beta_{1}, \beta_{2}, \beta_{3}, \beta_{4}, 1_{F}\right\}, \widetilde{\mho}_{2}=\left\{0_{\Delta}, \alpha_{1}, \alpha_{2}, \alpha_{3}, 1_{\Delta}\right\}$. Let $\psi: F \rightarrow \Delta$ be an identity maping. Then $\psi$ is f.ma.e-pc but not f.e-pc(resp. f.e-c) map because for the Fe-PO set $\alpha_{2}$ on $\Delta$, $\psi^{-1}\left(\alpha_{2}\right)=\alpha_{2}$ which is not a Fe-O set on $F$. In Example 3.2, $\psi$ is f.e-pc(resp. f.e-c) but not f.ma.e-pc.

Remark 3.22 Fuzzy minimal $e$-p.continuous and f.ma.e-pc are independent of each other.

Example 3.23 In Example 3.17, " $\psi$ is f.mi.e-pc map but it is not f.ma.e-pc map. From Example III, $\psi$ is f.ma.e-pc map but it is not f.mi.e-pc map."

Theorem 3.24 Let $F$ and $\Delta$ be ftss. A map $\psi: F \rightarrow \Delta$ is a f.e-pc iff the inverse image of each Fe-PC set in $\Delta$ is a fuzzy $e$-closed set in $F$.

Proof. Obvious.

Theorem 3.25 Let $A$ be a nonzero fuzzy subset of $F$. If $\psi: F \rightarrow \Delta$ is f.e-pc then the restriction map $\psi_{A}: A \rightarrow \Delta$ is a f.e-pc.

Proof. Let $\psi: F \rightarrow \Delta$ be a f.e-pc map and $A \subset F$. To prove $\psi_{A}$ is a f.e-pc. Let $\alpha$ be a Fe-PO set in $\Delta$. Since $\psi$ is f.e-pc, $\psi^{-1}(\alpha)$ is a Fe-O set in $F$. By the definition of relative topology $f_{A}^{-1}(\alpha)=A \wedge \psi^{-1}(\alpha)$. Therefore $A \wedge \psi^{-1}(\alpha)$ is a Fe-O set in $A$. Hence $\psi_{A}$ is a f.e-pc.

Remark 3.26 The composition of f.e-pc maps need not be f.e-pc.

Example 3.27 Let $F=\Delta=\Phi=\{a, b, c, d\}$ and the fuzzy sets $\beta_{1}=\frac{0.0}{a}+\frac{0.0}{b}+\frac{0.2}{c}+\frac{0.0}{d}, \beta_{2}=\frac{0.0}{a}+\frac{0.0}{b}+\frac{0.2}{c}+\frac{0.5}{d}$, $\beta_{3}=\frac{0.0}{a}+\frac{0.7}{b}+\frac{0.2}{c}+\frac{0.5}{d}$ and $\beta_{4}=\frac{0.3}{a}+\frac{0.7}{b}+\frac{0.2}{c}+\frac{0.5}{d}$ are defined as follows: Consider $\mathfrak{F}_{1}=\left\{0_{F}, \beta_{1}, \beta_{2}, 1_{F}\right\}, \mathfrak{F}_{2}=\left\{0_{\Delta}, \beta_{1}, \beta_{2}, \beta_{3}, 1_{\Delta}\right\}$ and $\widetilde{F}_{3}=\left\{0_{\Phi}, \beta_{1}, \beta_{3}, \beta_{4}, 1_{\Phi}\right\}$. Let $\psi: F \rightarrow \Delta$ and $\xi: \Delta \rightarrow \Phi$ be identity mappings. Then $\psi$ and $\xi$ are f.e-pc maps $\xi \circ \psi: F \rightarrow \Phi$ is not f.e-pc, since for the Fe-PO set $\beta_{3}$ in $\Phi, \psi^{-1}\left(\beta_{3}\right)=\beta_{3}$ which is not Fe-O set in $F$.

Theorem 3.28 If $\psi: F \rightarrow \Delta$ is f.e-c and $\xi: \Delta \rightarrow \Phi$ is f.e-pc. Then $\xi \circ: F \rightarrow \Phi$ is a f.e-pc.

Proof. Let $\tau_{1}$ be any Fe-PO set in $\Phi$. As $\xi$ is f.e-pc, $\xi^{-1}\left(\tau_{1}\right)$ is a Fe-O set in $\Delta$. Again since $\psi$ is f.e-c, $\psi^{-1}\left(\xi^{-1}\left(\tau_{1}\right)\right)=(\xi \circ \psi)^{-1}\left(\tau_{1}\right)$ is a Fe-O set in $F$. Hence $\xi \circ \psi$ is a f.e-pc.

Theorem 3.29 Let $F$ and $\Delta$ be ftss. A map $\psi: F \rightarrow \Delta$ is $*$-f.e-pc iff the inverse image of each fuzzy $e$-closed set in $\Delta$ is a Fe-PC set in $F$.

Proof. Obvious.

Remark 3.30 Let $F$ and $\Delta$ be fts. If $\psi: F \rightarrow \Delta$ is $*$-f.e-pc, then the restriction map $\psi_{A}: A \rightarrow \Delta$ need not be $*$-f.e-pc.

Example 3.31 Let $F=\Delta=\Phi=\{a, b, c\}$ and the fuzzy sets $\beta_{1}=\frac{0.7}{a}+\frac{0.0}{b}+\frac{0.0}{c}, \beta_{2}=\frac{0.7}{a}+\frac{0.3}{b}+\frac{0.0}{c}$ and $\beta_{3}=\frac{0.7}{a}+\frac{0.3}{b}+\frac{0.5}{c}$ are defined as follows: Consider $\mathfrak{F}=\left\{0_{F}, \beta_{1}, \beta_{2}, \beta_{3}, 1_{F}\right\}$ and $\mathfrak{F}_{1}=\left\{0_{\Delta}, \beta_{2}, 1_{\Delta}\right\}$. Let $\delta=\frac{0.0}{a}+\frac{0.3}{b}+\frac{0.9}{c}$ be a fuzzy set with $\mathfrak{F}_{\delta}=\left\{0_{\delta}, \beta_{4}, \beta_{5}, \beta_{6}, \delta\right\}$ where $\beta_{4}=\frac{0.0}{a}+\frac{0.3}{b}+\frac{0.0}{c}$ and $\beta_{5}=\frac{0.0}{a}+\frac{0.3}{b}+\frac{0.5}{c}$. Let $\psi: F \rightarrow \Delta$ be an identity map. Then $\psi$ is $*$-f.e-pc but $f_{\delta}: \widetilde{F}_{\delta} \rightarrow \Delta$ is not a $*$-f.e-pc, since for the Fe-O set $\beta_{2}$ in $\Delta, \psi^{-1}\left(\beta_{2}\right)=\beta_{2}$ which is not a Fe-PO set in $\widetilde{F}_{\delta}$.

Theorem 3.32 If $\psi: F \rightarrow \Delta$ and $\xi: \Delta \rightarrow \Phi$ is $*$-f.e-pc, then $\xi \circ \psi: F \rightarrow \Phi$ is a $*$-f.e-pc.

Proof. Let $\tau_{1}$ be any Fe-PO set in $\Phi$. As every Fe-PO set is a Fe-O set, $\xi^{-1}\left(\tau_{1}\right)$ is a Fe-PO set in $\Delta$. Again since $\psi$ is fuzzy $*$-f.e-pc, $\psi^{-1}\left(\xi^{-1}\left(\tau_{1}\right)\right)=(\xi \circ \psi)^{-1}\left(\tau_{1}\right)$ is a Fe-PO set in $F$. Hence $\xi \circ \psi$ is a $*$-f.e-pc.

Theorem 3.32 If $\psi: F \rightarrow \Delta$ is f.e-pc and $\xi: \Delta \rightarrow \Phi$ is $*$-f.e-pc, then $\xi \circ \psi: F \rightarrow \Phi$ is a f.e-pc(resp. f.e-c).

Proof. Let $\tau_{1}$ be any Fe-PO(resp. Fe-O ) set in $\Phi$. As every Fe-PO set is a Fe-O set, $\tau_{1}$ is a Fe-O set in $\Phi$. Since $\xi$ is a $*$-f.e-pc, $\xi^{-1}\left(\tau_{1}\right)$ is a Fe-PO set in $\Delta$. Again since $\psi$ is f.e-pc, $\psi^{-1}\left(\xi^{-1}\left(\tau_{1}\right)\right)=(\xi \circ \psi)^{-1}\left(\tau_{1}\right)$ is a Fe-O set in $F$. Hence $\xi \circ \psi$ is f.e-pc(resp. f.e-c) map.

Theorem 3.34 A map $\psi: F \rightarrow \Delta$ is f.e-p.i iff the inverse image of each fuzzy are $e$-paraclosed set in $\Delta$ is a Fe-PC set in $F$. Proof. Straightforward.

Remark 3.35 If $\psi: F \rightarrow \Delta$ is f.e-p.i. Then the restriction map $\psi_{A}: A \rightarrow \Delta$ need not be f.e-p.i. 
Example 3.36 In Example 3.2, let $\delta=\frac{0.0}{a}+\frac{0.0}{b}+\frac{0.6}{c}$ be a fuzzy set with $\mathfrak{\mho}_{\delta}=\left\{0_{\delta}, \beta_{4}, \delta\right\}$ where $\beta_{4}=\frac{0.0}{a}+\frac{0.0}{b}+\frac{0.5}{c}$. Let $\psi: F \rightarrow \Delta$ be an identity map. Then $\psi$ is f.e-p.i but $f_{\delta}: \mathfrak{\mho}_{\delta} \rightarrow \Delta$ is not a f.e-p.i, since for the Fe-PO set $\beta_{2}$ in $\Delta, \psi^{-1}\left(\beta_{2}\right)=\beta_{2}$ which is not a Fe-PO set in $\mathfrak{\mho}_{\delta}$.

Theorem 3.37 If $\psi: F \rightarrow \Delta$ is f.e-pc and $\xi: \Delta \rightarrow \Phi$ is f.e-p.i, then $\xi \circ \psi: F \rightarrow \Phi$ is a f.e-pc.

Proof. Let $\tau_{1}$ be a Fe-PO set in $\Phi$. As $\xi$ is a f.e-p.i $\xi^{-1}\left(\tau_{1}\right)$ is a Fe-PO set in $\Delta$. Again since $\psi$ is f.e-pc, $\psi^{-1}\left(\xi^{-1}\left(\tau_{1}\right)\right)=(\xi \circ \psi)^{-1}\left(\tau_{1}\right)$ is a Fe-O set in $F$. Hence $\xi \circ \psi$ is f.e-pc.

Theorem 3.38 If $\psi: F \rightarrow \Delta$ and $\xi: \Delta \rightarrow \Phi$ are f.e-p.i, then $\xi \circ \psi: F \rightarrow \Phi$ is a f.e-p.i.

Proof. Let $\tau_{1}$ be a Fe-PO set in $\Phi$. Since $\xi$ is a f.e-p.i $\xi^{-1}\left(\tau_{1}\right)$ is a Fe-PO set in $\Delta$. Again since $\psi$ is f.e-p.i, $\psi^{-1}\left(\xi^{-1}\left(\tau_{1}\right)\right)=$ $(\xi \circ \psi)^{-1}\left(\tau_{1}\right)$ is a Fe-PO set in $F$. Hence $\xi \circ \psi$ is f.e-pc.

Theorem 3.39 If $\psi: F \rightarrow \Delta$ is $*$-f.e-pc and $\xi: \Delta \rightarrow \Phi$ is f.e-p.i. Then $\xi \circ \psi: F \rightarrow \Phi$ is a f.e-p.i.

Proof. Let $\tau_{1}$ be a Fe-PO set in $\Phi$. As $\xi$ is a f.e-p.i, $\xi^{-1}\left(\tau_{1}\right)$ is a Fe-PO set in $\Delta$. Since every Fe-PO set is a Fe-O set, we have $\xi^{-1}\left(\tau_{1}\right)$ is a Fe-O set in $\Delta$. Again since $\psi$ is $*$-f.e-pc, $\psi^{-1}\left(\xi^{-1}\left(\tau_{1}\right)\right)=(\xi \circ \psi)^{-1}\left(\tau_{1}\right)$ is a Fe-PO set in $F$. Hence $\xi \circ \psi$ is f.e-p.i.

Theorem 3.40 If $\psi: F \rightarrow \Delta$ is f.e-p.i and $\xi: \Delta \rightarrow \Phi$ is $*$-f.e-pc, then $\xi \circ \psi: F \rightarrow \Phi$ is a f.e-p.i.

Proof. Let $\tau_{1}$ be a Fe-PO set in $\Phi$. As every Fe-PO set is a Fe-O set, $\tau_{1}$ is a Fe-O set in $\Phi$ Since $\xi$ is a f.e-pc, $\xi^{-1}\left(\tau_{1}\right)$ is a Fe-PO set in $\Delta$. Again Since $\psi$ is f.e-p.i, $\psi^{-1}\left(\xi^{-1}\left(\tau_{1}\right)\right)=(\xi \circ \psi)^{-1}\left(\tau_{1}\right)$ is a Fe-PO set in $F$. Hence $\xi \circ \psi$ is f.e-p.i mapping.

Theorem 3.41 A map $\psi: F \rightarrow \Delta$ is f.mi. f.e-pc iff the inverse image of each FMAe-C set in $\Delta$ is a Fe-PC set in $F$.

Proof. Obvious.

Remark 3.42 The composition of f.mi.e-pc maps need not be a f.mi.e-pc.

Example 3.43 Let $F=\Delta=\Phi=\{a, b, c, d\}$ and the fuzzy sets $\tau_{1}=\frac{0.0}{a}+\frac{0.0}{b}+\frac{0.2}{c}+\frac{0.4}{d}, \tau_{2}=\frac{0.0}{a}+\frac{0.7}{b}+\frac{0.2}{c}+\frac{0.4}{d}$, $\tau_{3}=\frac{0.2}{a}+\frac{0.7}{b}+\frac{0.2}{c}+\frac{0.4}{d}$ and $\tau_{4}=\frac{0.3}{a}+\frac{0.7}{b}+\frac{0.2}{c}+\frac{0.4}{d}$ are defined as follows: Consider $\mathfrak{F}_{1}=\left\{0_{F}, \tau_{1}, \tau_{2}, \tau_{3}, 1_{F}\right\}, \mathfrak{F}_{2}=\left\{0_{\Delta}, \tau_{2}, \tau_{3}, \tau_{4}, 1_{\Delta}\right\}$ and $\widetilde{\mho}_{3}=\left\{0_{\Phi}, \tau_{3}, \tau_{4}, 1_{\Phi}\right\}$. Let $\psi: F \rightarrow \Delta$ and $\xi: \Delta \rightarrow \Phi$ be identity mappings. Then $\psi$ and $\xi$ are f.mi.e-pc maps $\xi \circ \psi: F \rightarrow \Phi$ is not f.mi.e-pc, since for the FMIe-O set $\tau_{3}$ in $\Phi, \psi^{-1}\left(\tau_{3}\right)=\tau_{3}$ which is not Fe-PO set in $F$.

Theorem 3.44 If $\psi: F \rightarrow \Delta$ is f.e-p.i and $\xi: \Delta \rightarrow \Phi$ is f.mi.e-pc, then $\xi \circ \psi: F \rightarrow \Phi$ is a f.mi.e-pc.

Proof. Let $\eta$ be a FMIe-O set in $\Phi$. As $\xi$ is f.mi.e-pc, $\xi^{-1}(\eta)$ is a Fe-PO set in $\Delta$. Again since $\psi$ is f.e-p.i, $\psi^{-1}\left(\xi^{-1}(\eta)\right)=(\xi \circ \psi)^{-1}(\eta)$ is a Fe-PO set in $F$. Hence $\xi \circ \psi$ is f.mi.e-pc map.

Theorem 3.45 If $\psi: F \rightarrow \Delta$ is f.e-pc and $\xi: \Delta \rightarrow \Phi$ is f.mi.e-pc, then $\xi \circ \psi: F \rightarrow \Phi$ is a f.mi.e-pc.

Proof. Let $\eta$ be a FMIe-O set in $\Phi$. Since $\xi$ is f.mi.e-pc, $\xi^{-1}(\eta)$ is a Fe-PO set in $\Delta$. Again since $\psi$ is f.e-pc, $\psi^{-1}\left(\xi^{-1}(\eta)\right)=$ $(\xi \circ \psi)^{-1}(\eta)$ is a Fe-O set in $F$. Hence $\xi \circ \psi$ is f.mi.e-pc mapping.

Theorem 3.46 If $\psi: F \rightarrow \Delta$ is f.e-p.i and $\xi: \Delta \rightarrow \Phi$ is $*$-f.e-pc, then $\xi \circ \psi: F \rightarrow \Phi$ is a f.mi.e-pc.

Proof. Let $\eta$ be a FMIe-O set in $\Phi$. As every f.mi. $e$-open set is a Fe-O set, $\eta$ is an $e$-open set in $\Phi$. Since $\psi$ is $*$-f.e-pc, $\xi^{-1}(\eta)$ is a Fe-PO set in $\Delta$. Again since $\psi$ is f.e-p.i $\psi^{-1}\left(\xi^{-1}(\eta)\right)=(\xi \circ \psi)^{-1}(\eta)$ is a Fe-PO set in F. Hence $\xi \circ \psi$ is f.mi.e-pc.

Theorem 3.47 Let $F$ and $\Delta$ be fts. A map $\psi: F \rightarrow \Delta$ is f.ma.e-pc iff the inverse image of each FMIe-C set in $\Delta$ is a Fe-PC set in $F$.

Proof. Sraightforward.

Remark 3.48 The composition of f.ma.e-pc maps need not be a f.ma.e-pc.

Example 3.49 Let $F=\Delta=\Phi=\{a, b, c, d\}$ and the fuzzy sets $\tau_{1}=\frac{0.0}{a}+\frac{0.1}{b}+\frac{0.0}{c}+\frac{0.0}{d}, \tau_{2}=\frac{0.0}{a}+\frac{0.1}{b}+\frac{0.7}{c}+\frac{0.0}{d}$, $\tau_{3}=\frac{0.0}{x}+\frac{0.1}{y}+\frac{0.7}{z}+\frac{0.2}{w}$ and $\tau_{4}=\frac{0.3}{x}+\frac{0.1}{y}+\frac{0.7+}{z}+\frac{0.2}{w}$ are defined as follows: Consider $\mathfrak{F}_{1}=\left\{0_{F}, \tau_{2}, \tau_{3}, \tau_{4}, 1_{F}\right\}, \mathfrak{F}_{2}=\left\{0_{\Delta}, \tau_{1}, \tau_{2}, \tau_{3}, 1_{\Delta}\right\}$ and $\widetilde{\mho}_{3}=\left\{0_{\Phi}, \tau_{1}, \tau_{2}, 1_{\Phi}\right\}$. Let $\psi: F \rightarrow \Delta$ and $g: \Delta \rightarrow \Phi$ be identity mappings. Then $\psi$ and $\xi$ are f.ma.e-pc maps $\xi \circ \psi: F \rightarrow \Phi$ is not f.ma.e-pc, since for the FMAe-O set $\tau_{2}$ in $\Phi, \psi^{-1}\left(\tau_{2}\right)=\tau_{2}$ which is not Fe-PO set in $F$.

Theorem 3.50 If $\psi: F \rightarrow \Delta$ is f.e-p.i and $\xi: \Delta \rightarrow \Phi$ is f.ma.e-pc, hen $\xi \circ \psi: F \rightarrow \Phi$ is a f.ma.e-pc.

Proof. Let $\gamma$ be a FMAe-O set in $\Phi$. Since $\xi$ is f.ma.e-pc, $\xi^{-1}(\gamma)$ is a Fe-PO set in $\Delta$. Again since $\psi$ is f.e-p.i, $\psi^{-1}\left(\xi^{-1}(\gamma)\right)=$ $(\xi \circ \psi)^{-1}(\gamma)$ is a Fe-PO set in $F$. Hence $\xi \circ \psi$ is f.ma.e-pc.

Theorem 3.51If $\psi: F \rightarrow \Delta$ is f.e-pc and $\xi: \Delta \rightarrow \Phi$ is f.ma.e-pc, then $\xi \circ \psi: F \rightarrow \Phi$ is a f.ma.e-c.

Proof. Let $\gamma$ be a FMAe-O set in $\Phi$. Since $\xi$ is f.ma.e-pc, $\xi^{-1}(\gamma)$ is a Fe-PO set in $\Delta$. Again since $\psi$ is f.e-pc, $\psi^{-1}\left(\xi^{-1}(\gamma)\right)=$ $(\xi \circ \psi)^{-1}(\gamma)$ is a Fe-O set in $F$. Hence $\xi \circ \psi$ is f.ma.e-c.

Theorem 3.52 If $\psi: F \rightarrow \Delta$ is f.e-p.i and $\xi: \Delta \rightarrow \Phi$ is $*$-f.e-pc, then $\xi \circ \psi: F \rightarrow \Phi$ is a f.ma.e-pc. 
Proof. Let $\gamma$ be a FMAe-O set in $\Phi$. Since every FMAe-O set is a Fe-O set, $\gamma$ is a Fe-O set in $\Phi$. Since $\xi$ is $*$-f.e-pc, $\xi^{-1}(\gamma)$ is a Fe-PO set in $\Delta$. Again since $\psi$ is f.e-p.i, $\psi^{-1}\left(\xi^{-1}(\gamma)\right)=(\xi \circ \psi)^{-1}(\gamma)$ is a Fe-PO set in F. Hence $\xi \circ \psi$ is f.ma.e-pc.

\section{Conclusion}

The notion of fuzzy $e$-open sets is remarkable one. By means of this, fuzzy $e$-paraopen set introduced and studied. Also various fuzzy mappings and comparisons with appropriate examples investigated.

\section{ACKNOWLEDGMENT}

Authors are thankful to the referees for valuable suggestions and comments for improving manuscript.

\section{REFERENCES}

[1] Ajoy Mukherjee and Kallol Bhandhu Bagchi, On mean open set and mean closed sets, Kyungpook Math. J. 56(2016), $1259-1265$.

[2] C. L. Chang, Fuzzy topological spaces, J. Math. Anal. Appl., 24 (1968), 182-190.

[3] B. M. Ittanagi and R. S. Wali, On fuzy minimal open and fuzzy maximal open sets in fuzzy topological spaces, International J. of Mathematical Sciences and Applications,1(2),2011.

[4] B. M. Ittanagi and S. S. Benchalli, On paraopen sets and maps in topological spaces, Kyungpook Math. J., 56(1)(2016), 301-310.

[5] F. Nakaoka and N. Oda, Some Properties of Maximal Open Sets, International Journal of Mathematics and Mathematical Sciences, 21(2003), 1331-1340.

[6] F. Nakaoka and N. Oda, Minimal closed sets and maximal closed sets, International Journal of Mathematics and Mathematical Sciences, (2006), 1-8.

[7] M. Sankari, S. Durai raj and C. Murugesan, Fuzzy Minimal and Maximal e-Open Sets(Submitted).

[8] V. Seenivasan and K. Kamala, Fuzzy e-continuity and fuzzy e-open sets, Annals of Fuzzy Mathematics and Informatics, 8(1)(2014),141- 148.

[9] Supriti Saha, Fuzzy 8-continuous mappings, J. Math. Anal. Appl. 126 (1987) 130-142.

[10] L. A. Zadeh, Fuzzy sets, Information and control 8 (1965), 338-353.

M. Sankari received M.Phil., from Manonmaniam Sundaranar University in 1992 and received Ph.D. from Mother Tresa University in 2014. Her research interests include Applications of Fuzzy Sets, Topology and Fuzzy Topology.

C. Murugesan received M.Sc., from Manonmaniam Sundaranar University in 2014 and currently pursuing Ph.D., from Manonmaniam Sundaranar University, Tirunelvelli. 